\title{
Efecto de los costos de producción en el mercado de naranja en Veracruz, 1980-2018
}

\section{Production Costs Effect on the Orange Market in Veracruz, 1980-2018}

\author{
Fidel Bautista Mayorga* (D) https://orcid.org/0000-0003-1480-0239 \\ Ester Reyes Santiago** (D) https://orcid.org/0000-0003-0076-0563
}

\section{Resumen}

El objetivo de este artículo es determinar los factores que afectan el mercado de la naranja en Veracruz y analizar los efectos de los costos de producción en dicho mercado. En la metodología se utilizó la estimación de un modelo de ecuaciones simultáneas, usando datos anuales de 1980 a 20I8. Los resultados indican que los costos de producción no deben aumentar, porque dicho incremento ocasionaría efectos negativos en el mercado de la naranja. Se recomienda al gobierno federal ampliar el programa de fertilizantes a más cultivos de cítricos y que el aumento en el salario mínimo sea moderado, porque así se incentivan efectos positivos en el mercado, pues aumentan la producción y el precio medio rural.

Palabras clave: costos de producción; mercado de naranja; ecuaciones simultáneas; programa de fertilizantes; Veracruz.

\section{Abstract}

The purpose of this article is to determine the factors that affect the orange market in Veracruz and to analyze the effects of production costs on that market. The methodology used the estimation of simultaneous equations model, with annual data from 1980 to 2018. Results show that production costs should not increase, because the rise causes negative effects on the orange market. The conclusion leads to recommend the federal government's fertilizer program expansion to more crops, such as citrus. Another recommendation is to ask for a moderate minimum wage increase, inasmuch as it will encourage positive effects on the market to increase production and rural average price.

Keywords: production costs; orange market; simultaneous equations; fertilizer program; Veracruz.

Cómo citar: Bautista Mayorga, F., y Reyes Santiago, E. (2020). Efecto de los costos de producción en el mercado de naranja en Veracruz, 1980-20 I. región y sociedad, 32, el 294. doi: I0.22 I98/rys2020/32/I294

\footnotetext{
* Colegio de Postgraduados, km. 36.5, carretera México-Texcoco, C. P. 56230,

Montecillo, Estado de México, México. Correo electrónico: bautista.fidel@colpos.mx

** Autora para correspondencia. Colegio de Postgraduados, km. 36.5, carretera México-Texcoco, C. P. 56230, Montecillo, Estado de México, México. Correo electrónico: reyes.ester@colpos.mx
} 


\section{Introducción}

La citricultura es una de las actividades de importancia económica en México, en especial para los estados productores. Datos del Servicio de Información Agroalimentaria y Pesquera (SIAP, 2019) revelan que el valor de la producción en esta actividad supera los 26187.9 millones de pesos. Es una fuente generadora de empleos, pues en esta actividad participan alrededor de 67 mil productores, genera 13.9 millones de jornales, 46 mil empleos directos, 139 mil empleos indirectos y deja una derrama económica de 10335 millones de pesos (Caamal, Jerónimo y Pat, 2016; Cárdenas, 2006). En 2018 el valor de las exportaciones de la naranja en fruta, jugo, esencia y cáscara fue de 28, 469, 51 y 11 millones de dólares, respectivamente (Panorama Agroalimentario, 2019).

En la tabla 1 se observa que la naranja es el cítrico de mayor importancia en México. Participa con $58.3 \%$ de la superficie sembrada, $59 \%$ en la producción y $38.9 \%$ del valor de la producción. Además, es el fruto más popular en la dieta de los mexicanos por ser una fuente de vitamina C (SIAP, 2019).

Tabla 1. Estructura productiva de los cítricos en México, 2018

\begin{tabular}{|c|r|r|r|r|}
\hline Cítricos & $\begin{array}{c}\text { Superficie } \\
\text { sembrada } \\
\text { (ha) }\end{array}$ & $\begin{array}{c}\text { Superficie } \\
\text { cosechada } \\
\text { (ha) }\end{array}$ & $\begin{array}{c}\text { Producción } \\
\text { (t) }\end{array}$ & \multicolumn{1}{|c|}{$\begin{array}{l}\text { Valor de la } \\
\text { producción } \\
\text { (miles de pesos) }\end{array}$} \\
\hline Naranja & 339759.43 & 326689.01 & 4737990.29 & 10186462.2 \\
\hline Limón & 200669.05 & 174406.43 & 2533176.15 & 13873685.8 \\
\hline Mandarina & 21675.82 & 20829.52 & 300065.23 & 813495.96 \\
\hline Toronja & 20221.53 & 18823.31 & 459609.93 & 1314218.9 \\
\hline Total & 582325.83 & 540748.27 & 8030841.6 & 26187862.8 \\
\hline Cítricos & $\begin{array}{r}\text { Superficie } \\
\text { sembrada } \\
\text { (porcentaje) }\end{array}$ & $\begin{array}{r}\text { Superficie } \\
\text { cosechada } \\
\text { (porcentaje) }\end{array}$ & $\begin{array}{r}\text { Producción } \\
\text { (porcentaje) }\end{array}$ & $\begin{array}{r}\text { Valor de la } \\
\text { producción } \\
\text { porcentaje) }\end{array}$ \\
\hline Naranja & 58.3 & 60.4 & 59.0 & 38.9 \\
\hline Limón & 34.5 & 32.3 & 31.5 & 5.1 \\
\hline Mandarina & 3.7 & 3.9 & 3.7 & 5.0 \\
\hline Toronja & 3.5 & 3.5 & 5.7 & 100.0 \\
\hline Total & 100.0 & 100.0 & 100.0 & \\
\hline
\end{tabular}

Fuente: elaboración propia con datos del SIAP (2019).

En la tabla 2 se presentan los principales estados productores de naranja en México. Sin duda, Veracruz es el líder en la superficie sembrada, en producción y en valor total de ésta, Aporta 50\%, 53\% y 46\%, respectivamente. Otros estados que destacan por su nivel de producción son Tamaulipas, San Luis Potosí, Nuevo León, Puebla, Sonora y Yucatán, que concentran 39.2\% de ésta (SIAP, 2019). 


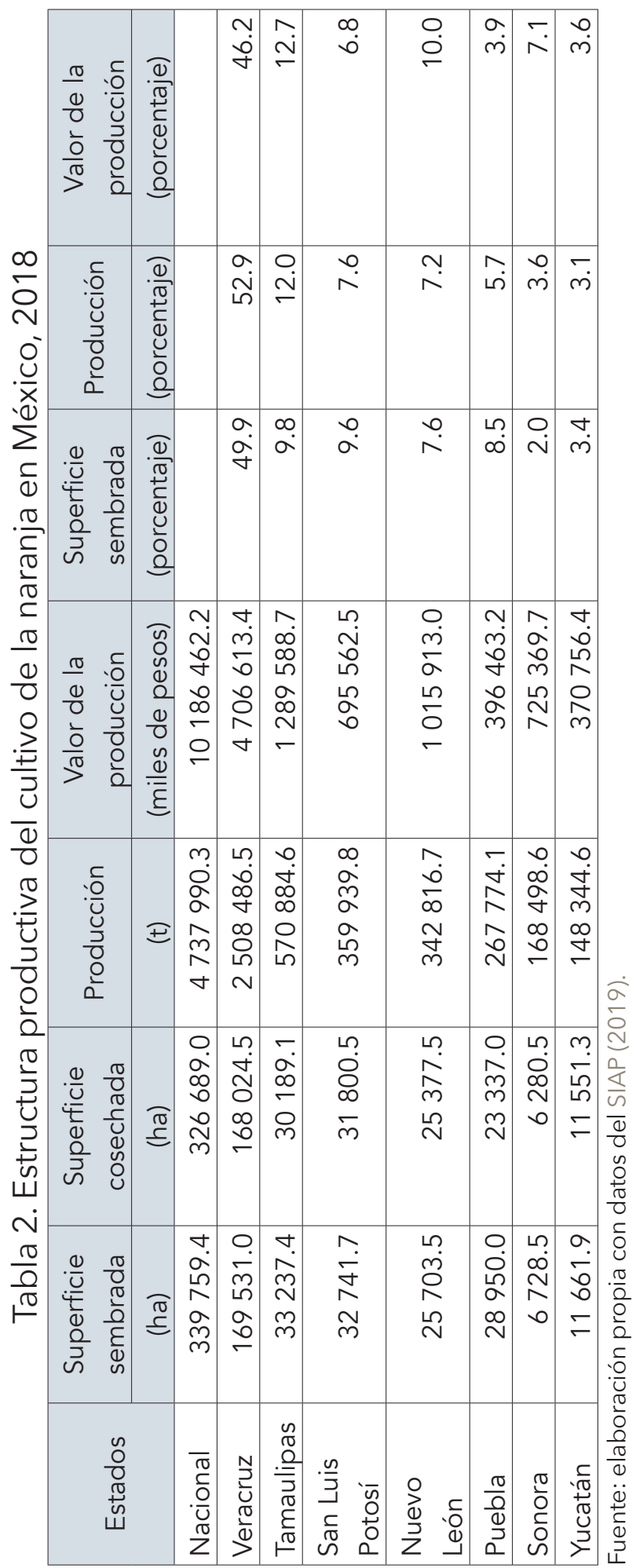


Los costos de producción se han convertido en una variable de gran trascendencia para los productores agrícolas, ya que son la base fundamental para planificar, controlar y tomar decisiones (Ochoa, 2015). En el caso de los cítricos, se incluye la mano de obra y el precio de los fertilizantes como costos de producción (Caamal et al., 2016; Soler y Hernández, 2005).

El cultivo de la naranja en el estado de Veracruz es intensivo en el uso de mano de obra, sobre todo en la cosecha, tanto así que en el periodo de la explotación del cultivo es insuficiente la fuerza de trabajo local y se recurre a los migrantes. Se estima que se necesitan alrededor de 70 jornales por hectárea (Barrón y Hernández, 2014). Las tareas que implica la citricultura en Veracruz son variadas; entre ellas, atender las labores relacionadas con el cultivo de la naranja (rastra, deshierbe, cajeteo, ${ }^{1}$ poda), llevar el control fitosanitario, fertilización (suelo y foliar) (Secretaría de Agricultura, Ganadería, Desarrollo Rural, Pesca y Alimentación [SAGARPA], 2015). Datos recientes indican que los costos de producción de la mano de obra y el fertilizante en el cultivo de naranja representan $15.5 \%$ y $45.4 \%$ del total de los costos, respectivamente (Fideicomisos Instituidos en Relación con la Agricultura [FIRA], 2018). Dado lo anterior, es importante analizarlos, porque tienen un peso significativo en la estructura total de los costos y determinan la producción de los cultivos agrícolas, tal como se constata en los trabajos de Almaguer-Vargas y Ayala-Garay (2014); Aznar, Pérez y Galdeano (2015); Barrera-Rodríguez, Jaramillo-Villanueva, Escobedo-Garrido y Herrera-Cabrera (2011); Franco, Leos, Salas, Acosta y García (2018); López-Hernández, Garza-Bueno, Cruz-Galindo y Nieto-Ángel (2019); Molina (2016); Pat-Fernández, Caamal-Cauich, Jerónimo-Ascencio y Mendoza-Tornez (2015); Ramírez, Figueroa y Espinoza (2015) y Rebollar, Hernández, Guzmán, Morales y Rebollar (2014).

El pago que perciben los jornaleros de naranja en Veracruz es de dos salarios mínimos. Esto puede traer consecuencias en el mercado de naranja, porque los salarios aumentan los costos de producción hasta igualar el precio de venta de la fruta y el productor se queda con nulas ganancias (Barrón y Hernández, 2014; Tierra Fértil, 2019). La problemática que aqueja a los productores de naranja en Veracruz es que los precios de venta son muy bajos, lo cual trae como consecuencia que al considerar los costos de producción, de acarreo y de comercialización, la ganancia sea nula (Tierra Fértil, 2019). Ante esto, es relevante analizar los principales factores que afectan el mercado de la naranja en la entidad, con el fin de brindar recomendaciones de política a los tomadores de decisiones y de esta manera ayudar a mejorar la situación que prevalece en la entidad.

Los fertilizantes son uno de los insumos agrícolas indispensables para nutrir los cultivos y mejorar su rendimiento y por ende aumentar la producción. A partir de 2019, la Secretaría de Agricultura y Desarrollo Rural (SADER) emprendió el programa de fertilizantes con cobertura nacional, el cual tiene como objetivo principal incentivar la producción de cultivos prioritarios (maíz, frijol y arroz) para localidades con altos grados de marginación (Diario Oficial de la Federación, 2019).

1 Cajeteo se refiere a hacer hoyos en la tierra para después plantar. 
Otra política llevada a cabo por el gobierno federal de México ha sido incrementar en términos nominales el salario mínimo: primero pasó de 88.36 a 102.68 pesos diarios (de diciembre de 2018 a enero de 2019) y en enero de 2020 incrementó en $20 \%$ más a escala nacional (al pasar de 102.68 a 123 pesos diarios) y $5 \%$ en la zona libre de la frontera norte (al pasar de 176.72 a 185.56 pesos diarios) (Hernández, 2019).

Por otra parte, la aplicación de fertilizantes es importante para obtener buena producción de naranja. En Veracruz 30\% de los agricultores lleva a cabo esta práctica, el resto logra obtener una producción gracias a una buena floración del cultivo. Aplicar fertilizante puede aumentar el rendimiento promedio de 10 a 30 toneladas por hectárea. Incluso si hay buen suelo podría llegar hasta 50 toneladas. A pesar de que la citricultura tiene varios problemas, como falta de fertilización, enfermedades, plagas e insuficiencia en mano de obra en épocas de cosecha, tiene buen futuro si los productores adoptan la cultura de invertir más en las huertas (Moreno, 2017). El pago de la mano de obra (salarios mínimos) y los fertilizantes tienen un peso considerable en la estructura de los costos de producción. Sería conveniente que el gobierno subsidiara el fertilizante en Veracruz, pues bajaría el costo de producción y en consecuencia ésta aumentaría.

Considerando la importancia económica y social que tiene el cultivo de la naranja en Veracruz, el principal estado productor de este cítrico, el presente artículo persigue los siguientes objetivos: 1 ) determinar los factores que afectan el mercado de la naranja en Veracruz y 2) analizar el efecto de los costos de producción (política del gobierno actual, aumento del salario mínimo y subsidio en el fertilizante) sobre el mercado de la naranja en el estado de Veracruz, mediante escenarios de predicción. Se plantearon dos hipótesis. La primera es que los factores determinantes de la demanda en dicha entidad son la población y el ingreso per cápita y, por otro lado, los de la oferta son la temperatura y los costos de producción. La segunda es que ante incrementos en los costos de producción, la oferta de naranja disminuye, y viceversa.

\section{Materiales y métodos}

El modelo

Para cumplir con los objetivos, se utilizó un modelo de ecuaciones simultáneas compuesto por una ecuación de oferta, una de demanda, tres de transmisión de precios y una identidad de saldo de comercio exterior. Se emplearon datos anuales de 1980 a 2018. El modelo contempló aspectos de la teoría económica y evidencia empírica sobre el mercado de la naranja en Veracruz. De acuerdo con Gujarati y Porter (2010), la estimación de los coeficientes del modelo fue por mínimos cuadrados en dos etapas (MC2E) debido a la condición de sobreidentificación de las ecuaciones. Dicha estimación se realizó a través del paquete estadístico Statistical Analysis System Institute (SAS, 2013). A continuación se presenta el modelo: 


$$
\begin{aligned}
& Q P N=\beta_{0}+\beta_{1} P N R 5 L+\beta_{2} P L R 4 L+\beta_{3} P M R 5 L+\beta_{4} P T R 5 L+\beta_{5} P M Z R L+\beta_{6} P F R \\
& +\beta_{7} P C H R L+\beta_{8} P S R L+\beta_{9} S M G R+\beta_{10} F E R T R+\beta_{11} P P+\beta_{12} T E M P+\beta_{13} Q P N L+\varepsilon_{1}
\end{aligned}
$$

Donde: QPN es la cantidad producida de naranja, en el periodo actual, en toneladas (t); PNR5L es el precio medio rural real de la naranja, con cinco periodos de rezago, en pesos por tonelada $(\$ / \mathrm{t}) ; P L R 4 L$ es el precio medio rural real del limón, con cuatro periodos de rezago, en $\$ / \mathrm{t} ; P M R 5 L$ es el precio medio rural real de la mandarina, con cinco periodos de rezago, en $\$ / t$; PTR5L es el precio medio rural real de la toronja, con cinco periodos de rezago, en $\$ / \mathrm{t} ;$ PMZRL es el precio medio rural real del maíz, con un periodo de rezago, en $\$ / \mathrm{t}$; $P F R L$ es el precio medio rural real del frijol, con un periodo de rezago, en $\$ / t ; P C H R L$ es el precio medio rural real del chile, con un periodo de rezago, en $\$ / \mathrm{t}$; PSRL es el precio medio rural real de la sandía, con un periodo de rezago, en $\$ / \mathrm{t} ; S M G R$ es el salario mínimo general real, en el periodo actual, en pesos diarios; FERTR es el precio promedio real de los fertilizantes ${ }^{2}$ en el periodo actual, en $\$ / \mathrm{t} ; P P$ es la precipitación media anual de Veracruz, en el periodo actual, en milímetros $(\mathrm{mm})$; TEMP es la temperatura promedio anual de Veracruz, en el periodo actual, en grados Celsius $\left({ }^{\circ} \mathrm{C}\right)$; QPNL es la cantidad producida de naranja, con un periodo de rezago, en toneladas.

Los términos del error aleatorio están expresados al final de cada ecuación $\left(\varepsilon_{1}, \ldots, \varepsilon_{5}\right)$.

$Q D N=\alpha_{0}+\alpha_{1} P C N R+\alpha_{2} P C L R+\alpha_{3} P C T R+\alpha_{4} I N G P R+\alpha_{5} P O B+\alpha_{6} Q D N L+\varepsilon_{2}$

Donde: QDN es la cantidad demandada de naranja en Veracruz, en el periodo actual, en t; $P C N R$ es el precio promedio real al consumidor de naranja, en el periodo actual, en $\$ / \mathrm{t}$; $P C L R$ es el precio promedio real al consumidor del limón, en el periodo actual, en $\$ / \mathrm{t} ;$ PCTR es el precio promedio real al consumidor de la toronja, en el periodo actual, en $\$ / \mathrm{t}$; INGPR es el ingreso per cápita real, en el periodo actual, en miles de pesos; $P O B$ es la población mayor a cinco años en Veracruz, en el periodo actual, en personas; QDNL es la cantidad demandada de naranja en Veracruz, con un periodo de rezago, en $\mathrm{t}$.

$$
P N R=\mu_{0}+\mu_{1} P M N R+\mu_{2} P N R L+\varepsilon_{3}
$$

Donde: PNR es el precio medio rural real de la naranja, en el periodo actual, en $\$ / \mathrm{t}$; PMNR es el precio promedio real al mayoreo de la naranja en Veracruz, en el periodo actual, en $\$ / t$; PNRL es el precio medio rural real de la naranja, en un periodo de rezago, en $\$ / \mathrm{t}$.

2 Los fertilizantes que por lo general se usan en la naranja son urea, nitrato de amonio, sulfato de amonio, superfosfato triple y simple, fosfato diamónico y cloruro de potasio (SAGARPA, 2015). 
$P M N R=\mu_{0}+\mu_{1} Q P N+\mu_{2} Q D N+\mu_{3} P E N R+\mu_{4} P I N R+\mu_{5} P M N R L+\mu_{6} D+\varepsilon_{4}$

Donde: PENR es el precio real de exportación de la naranja de México, en el periodo actual, en $\$ / \mathrm{t}$; PINR es el precio real de importación de la naranja en México, en el periodo actual, en $\$ / \mathrm{t} ; P M N R L$ es el precio promedio real al mayoreo de la naranja en Veracruz, con un periodo de rezago, en $\$ / \mathrm{t} ; D$ es una variable de clasificación, donde $D=0$ cuando no se considera una economía abierta en México (1980-1994), y $D=1$ cuando sí se considera una economía abierta (1995-2018).

$$
P C N R=\mu_{0}+\mu_{1} P M N R+\mu_{2} P C N R L+\varepsilon_{5}
$$

Donde: PCNRL es el precio promedio real al consumidor de naranja, con un periodo de rezago, en $\$ / t$.

$S C E N=Q P N-Q D N$

Donde: SCEN es el saldo de comercio exterior de la naranja de Veracruz, en el periodo actual, en $t$.

En teoría, la oferta de un producto está determinada por el precio que recibe el productor, el de los bienes sustitutos o complementarios en la producción, el de los factores productivos o insumos, la tecnología, los impuestos o subsidios, el clima, la expectativa del productor (cantidad y precio futuro) y el número de competidores. Por el lado de la demanda, los factores que la determinan son el precio pagado por el consumidor, el de los bienes sustitutos o complementarios, la población, el ingreso, la expectativa del consumidor (cantidad y precio futuro), gustos y preferencias (Atucha y Gualdoni, 2018; Barkley y Barkley, 2013).

En términos empíricos, Gómez y Schwentesius (1997) y Hernández (2011) indican que en el estado de Veracruz y los estados vecinos asocian el maíz, el frijol, el chile y la sandía con el cultivo de la naranja, aunque no existen estudios serios sobre el impacto positivo o negativo en el desarrollo del cítrico. Puesto que están asociados, en este trabajo se decidió incorporarlos para determinar y aportar evidencia econométrica sobre el tipo de relación que existe entre estos cultivos (competitivo o asociado). Por el lado de la demanda, el limón y la toronja son cítricos representativos que se asocian con el consumo de la naranja y son cultivos que mantienen una relación funcional inversa; es decir, si aumenta el precio del limón y de la toronja, disminuye la demanda de naranja, o viceversa (López, Valdivia, Hernández y Romo, 2010).

Con base en lo anterior, los factores que determinaron la oferta de naranja en Veracruz fueron el precio de la naranja, del limón, de la mandarina, de la toronja, del salario, del fertilizante, la precipitación y la temperatura. La cantidad producida con un periodo de rezago se consideró como expectativa del productor. Del mismo modo, los factores que determinaron la demanda de naranja en dicha entidad son el precio al consumidor de ésta, del limón, de la 
toronja, el ingreso y la población. La cantidad demandada de naranja con un periodo de rezago se consideró como expectativa del consumidor.

Datos del modelo

La serie de los precios medios rurales de naranja, limón, mandarina, toronja, maíz, frijol, chile, sandía y la cantidad producida de naranja en Veracruz (de 1980 a 2018) provinieron del SIAP (2019). Debido a la no disponibilidad de datos, el precio promedio de los fertilizantes se obtuvo del Sistema Nacional de Información e Integración de Mercados (SNIIM, 2019a) para Veracruz (20002018), y para México (1980-1999), de la Statistics División of Food and Agriculture Organization of the United Nations (FAOSTAT, 2019a). El precio al mayoreo de la naranja (1998-2018) provino del SNIIM (2019b) y se estimaron los datos faltantes (1980-1997) a través de márgenes de comercialización. El dato del salario mínimo general (1980-2018) provino de la Comisión Nacional de Salarios Mínimos (CONASAMI, 2019). La serie de temperatura y precipitación se tomó del Servicio Meteorológico Nacional (SMN, 2019) (1985-2018) y de la Secretaría del Medio Ambiente y Recursos Naturales (SEMARNAT, 2019) (1980-1984). Los precios promedio al consumidor de naranja, limón y toronja (1999-2018) provinieron de la Procuraduría Federal del Consumidor (PROFECO) (C. M. Orozco, comunicación personal, 22 de marzo de 2019). Debido a la no disponibilidad de datos en las fuentes oficiales de México, los datos faltantes se estimaron a través de márgenes de comercialización de 1980 a 1998. Así mismo, la serie de la cantidad demandada de naranja en Veracruz (1980-2018) fue estimada de la siguiente manera: primero se calculó el consumo nacional aparente (CNA) de naranja per cápita (la producción más las importaciones, menos las exportaciones de naranja en México, y el resultado se divide entre la población de México), después se multiplica dicho CNA por la población anual de Veracruz (ponderador). La serie de población de Veracruz (de 1980 a 2018) se obtuvo del Consejo Nacional de Población (CONAPO, 2019), y la población de México (de 1980 a 2018), de la FAOSTAT (2019b). El ingreso per cápita se estimó con el producto interno bruto (PIB), y en cuanto a la población de Veracruz, los datos provinieron del Instituto Nacional de Estadística y Geografía (INEGI, 2019a). La serie de precios de exportación y de importación de la naranja (1980-2018) se obtuvo de la FAOSTAT (2019c). Cabe mencionar que estas unidades vienen expresadas en dólares y para convertirlas a pesos mexicanos fue necesario emplear el tipo de cambio Fixed Exchange Rate (FIX por sus siglas en inglés) promedio anual de 1980 a 2018, el cual se obtuvo del Banco de México (BANXICO, 2019). La serie de saldo de comercio exterior (de 1980 a 2018) fue estimada restándole a la cantidad producida la cantidad demandada de naranja en Veracruz. Para deflactar los datos monetarios, se usó el Índice Nacional de Precios al Productor y Consumidor, que se obtuvieron del INEGI (2019b).

Formulación del modelo

El modelo de ecuaciones simultáneas se puede expresar de manera matricial de la siguiente manera (Gujarati y Porter, 2010): 
$\Gamma Y_{t}+\beta X_{t}=E_{t}$

Donde: $Y_{t}$ es el vector de variables endógenas o dependientes del modelo; $X_{t}$ es el vector de variables exógenas del modelo, más la ordenada al origen; $\Gamma$ es la matriz de parámetros estructurales asociados a las variables endógenas; $B$ es la matriz de parámetros estructurales asociados a las variables exógenas; $E_{t}$ son los términos de errores aleatorios. Los vectores $Y_{t}$ y $E_{t}$ son de orden $m x$ 1 , donde $m$ es el número de variables endógenas del modelo, $\Gamma$ es una matriz cuadrada de $m \times m$, a su vez, $B$ es una matriz de $k+1 \times m$, donde $k$ es el número de variables exógenas y endógenas rezagadas del modelo, más la ordenada al origen; en general, $k$ puede o no ser igual a $m$. Para que el sistema esté completo, debe existir la inversa de $\Gamma$, es decir, $\Gamma$ debe ser una matriz no singular de orden $m$, para derivar el modelo reducido del sistema de la siguiente manera:

$$
Y_{t}=\pi X_{t}+V_{t}
$$

Donde: $\Pi$ es igual a - $\Gamma^{-1} B$ y es la matriz de parámetros de la forma reducida; $V_{t}$ es igual a $\Gamma^{-1} E_{t}$ es la matriz de los términos de error en la forma reducida.

Calibración del modelo y cálculo de elasticidades

Una vez resuelto el sistema de ecuaciones, se calibró el modelo. En otras palabras, se compararon los valores de las variables endógenas predichos por el modelo, con respecto a los valores observados de éstas. Este procedimiento resultó satisfactorio por la poca diferencia porcentual entre ambos valores.

Después se obtuvo la elasticidad precio y cruzadas de la oferta y la demanda, además de las elasticidades relacionadas con la transmisión de precios y el saldo de comercio exterior de la naranja en Veracruz. Se emplearon, para tal propósito, los valores de los parámetros estimados por el modelo de la forma estructural y restringida, así como el valor medio de las variables endógenas y exógenas observadas en los últimos tres años (2016-2018). Con base en Barkley y Barkley (2013) y Nicholson (2008), y a manera de ejemplo, se expresa la fórmula de la elasticidad:

$$
\epsilon_{P C N R}^{Q D N}=\frac{\frac{\Delta Q D N}{Q D N}}{\frac{\Delta P C N R}{P C N R}}=\frac{\Delta Q D N}{\Delta P C N R} *\left(\frac{P C N R}{Q D N}\right)=\frac{\partial Q D N}{\partial P C N R} *\left(\frac{P C N R}{Q D N}\right)=\alpha *\left(\frac{P C N R}{Q D N}\right)
$$

Donde: $\epsilon_{P C N R}^{O D N}$ es la elasticidad precio de demanda; es el valor del parámetro estimado por el modelo, que relaciona a la cantidad demandada respecto al precio (la pendiente), y lo que está entre paréntesis son los valores medios de las variables antes especificadas. Así, sucesivamente, se procedió para obtener las elasticidades por cada variable independiente en el modelo, en relación con la variable dependiente. 
Por último, se propusieron siete escenarios de política económica modificando algunos factores que determinan la producción de naranja en Veracruz, en específico los que tienen que ver con los costos de producción en dicha entidad (salario mínimo y precio del fertilizante). En todos los escenarios se usó el término ceteris paribus, que es un supuesto usado por los economistas en los modelos para mantener constantes diversas influencias externas, de modo que les permita analizar de manera más simplificada la relación precisa que se está estudiando (Nicholson, 2008).

\section{Resultados y discusión}

Resultados estadísticos

En las cinco ecuaciones, que se muestran en la tabla 3, se puede observar que el coeficiente de determinación varía entre 0.52 y 0.92 , lo cual indica una bondad de ajuste aceptable entre los datos predichos por el modelo y los datos observados. La prueba de $\mathrm{F}$ indica la significancia global de los parámetros considerados en el modelo con una probabilidad menor a 1\%, mientras que la prueba t de student (t) señala la significancia individual del valor de los parámetros, en la cual se puede percibir que la mayoría de los coeficientes son significativos, debido a que superan la unidad. La congruencia económica se valida por los signos que acompañan a las variables exógenas del modelo. Por último, los valores relacionados con el estadístico Durbin-Watson (DW) oscilan entre 0.76 y 2.08 . Éstos indican que no existe autocorrelación en cuatro de cinco ecuaciones.

Tabla 3. Resultados de la forma estructural del mercado de la naranja en Veracruz, 1980-2018

\begin{tabular}{|c|c|c|c|c|c|c|c|c|}
\hline Ecuación & & Intercepto & PNR5L & PLR4L & PMR5L & PTR5L & $P M Z R L$ & PFRL \\
\hline & Coeficiente & -2984160 & 128.2 & -15.3 & -22.5 & -10.7 & 21.2 & 9.8 \\
\hline & Error estándar & 2354555 & 48.0 & 15.6 & 21.6 & 18.5 & 66.5 & 11.7 \\
\hline & Razón t & -1.3 & 2.7 & -1.0 & -1.1 & -0.6 & 0.3 & 0.8 \\
\hline & Prob. (t) & 0.220 & 0.015 & 0.339 & 0.308 & 0.570 & 0.754 & 0.413 \\
\hline & & PCHRL & PSRL & $S M G R$ & FERT & $P P$ & TEMP & QPNL \\
\hline & Coeficiente & -4.9 & 78.3 & -6881.3 & -55.4 & -89.2 & 185271 & 0.7 \\
\hline & Error estándar & 10.3 & 29.8 & 2140.6 & 18.7 & 209.6 & 89953.8 & 0.2 \\
\hline & Razón t & -0.5 & 2.6 & -3.2 & -3.0 & -0.4 & 2.1 & 3.6 \\
\hline & Prob. (t) & 0.638 & 0.016 & 0.004 & 0.008 & 0.675 & 0.053 & 0.002 \\
\hline & \multicolumn{2}{|c|}{$\mathrm{R}^{2}=0.82$} & \multicolumn{2}{|c|}{ Prob $>F=0.0001$} & \multicolumn{2}{|c|}{$D W=1.97$} & & \\
\hline & \multicolumn{8}{|c|}{$\begin{array}{c}\text { QPN=2 } 984 \text { 160+128.2PNR5L15.3PLR4L22.5PMR5L10.7PTR5L+21.2PMZRL+9.8PFRL } \\
\text { 4.9PCHRL+78.3PSRL68 881.3SMGR55.4FERT89.2PP+185 271TEMP+0.7QPNL }\end{array}$} \\
\hline
\end{tabular}




\begin{tabular}{|c|c|c|c|c|c|c|c|c|}
\hline \multirow{7}{*}{ QDN } & & Intercepto & PCNR & $P C L R$ & PCTR & INGPR & $P O B$ & QDNL \\
\hline & Coeficiente & -7740.2 & -0.3 & 0.6 & -0.3 & 100.6 & 0.01 & 0.5 \\
\hline & Error estándar & 28927.1 & 0.6 & 0.5 & 0.3 & 130.6 & 0.01 & 0.2 \\
\hline & Razón t & -0.3 & -0.5 & 1.1 & -1.0 & 0.8 & 1.2 & 2.9 \\
\hline & Prob. (t) & 0.791 & 0.621 & 0.267 & 0.321 & 0.448 & 0.249 & 0.007 \\
\hline & \multicolumn{2}{|c|}{$\mathrm{R}^{2}=0.85$} & \multicolumn{2}{|c|}{ Prob $>F=0.0001$} & \multicolumn{2}{|c|}{$D W=1.62$} & & \\
\hline & \multicolumn{8}{|c|}{$\mathrm{QDN}=7$ 740.20.3PCNR+0.6PCLR-0.3PCTR+100.6INGPR+0.01POB+0.5QDNL } \\
\hline \multirow{7}{*}{ PNR } & & Intercepto & PMNR & PNRL & & & & \\
\hline & Coeficiente & 521.8 & 0.1 & 0.4 & & & & \\
\hline & Error estándar & 282.9 & 0.04 & 0.1 & & & & \\
\hline & Razón t & 1.8 & 2.5 & 3.9 & & & & \\
\hline & Prob. (t) & 0.0747 & 0.0174 & 0.0004 & & & & \\
\hline & \multicolumn{2}{|c|}{$\mathrm{R}^{2}=0.52$} & \multicolumn{2}{|c|}{ Prob $>F=0.0001$} & \multicolumn{2}{|c|}{$D W=2.08$} & & \\
\hline & \multicolumn{8}{|c|}{$P N R=521.8+0.1 P M N R+0.4 P N R L$} \\
\hline \multirow{7}{*}{ PMNR } & & Intercepto & QPN & QDN & PENR & PINR & PMNRL & $D$ \\
\hline & Coeficiente & 5734.6 & 0.01 & -0.2 & -0.2 & 0.3 & 0.3 & 890.2 \\
\hline & Error estándar & 3511.3 & 0.004 & 0.1 & 0.1 & 0.2 & 0.1 & 1825.6 \\
\hline & Razón t & 1.6 & 2.1 & -2.3 & -1.2 & 1.6 & 2.0 & 0.5 \\
\hline & Prob. (t) & 0.114 & 0.050 & 0.031 & 0.242 & 0.124 & 0.060 & 0.630 \\
\hline & \multicolumn{2}{|c|}{$\mathrm{R}^{2}=0.58$} & \multicolumn{2}{|c|}{ Prob $>F=0.0003$} & \multicolumn{2}{|c|}{$\mathrm{DW}=2.03$} & & \\
\hline & \multicolumn{8}{|c|}{$P M N R=5734.6+0.01 Q P N-0.2 Q D N 0.2 P E N R+0.3 P I N R+0.3 P M N R L 890.2 D$} \\
\hline \multirow{7}{*}{ PCNR } & & Intercepto & PMNR & PCNRL & & & & \\
\hline & Coeficiente & 231.3 & 2.1 & 0.1 & & & & \\
\hline & Error estándar & 758.8 & 0.1 & 0.1 & & & & \\
\hline & Razón t & 0.3 & 14.7 & 1.1 & & & & \\
\hline & Prob. $(\mathrm{t})$ & 0.763 & 0.0001 & 0.299 & & & & \\
\hline & \multicolumn{2}{|c|}{$\mathrm{R}^{2}=0.92$} & \multicolumn{2}{|c|}{ Prob $>F=0.0001$} & \multicolumn{2}{|c|}{$\mathrm{DW}=0.76$} & & \\
\hline & & PCNR & $.3+2.1 P$ & $\mathrm{MNR}+0$ & ${ }^{\circ} \mathrm{CNRL}$ & & & \\
\hline
\end{tabular}

Fuente: elaboración propia con datos de salida del modelo.

\section{Resultados de la forma reducida del modelo}

En la tabla 4 se muestran los resultados de la forma reducida restringida del modelo, que básicamente son las variables endógenas expresadas sólo por las variables exógenas del modelo. 
Tabla 4. Resultados de la forma reducida restringida del mercado de la naranja en Veracruz, 1980-2018

\begin{tabular}{|c|c|c|c|c|c|}
\hline Variables & \multicolumn{2}{|c|}{ Variables endógenas } & \multirow{2}{*}{$\begin{array}{l}\text { Variables } \\
\text { Exógenas }\end{array}$} & \multicolumn{2}{|c|}{ Variables endógenas } \\
\hline exógenas & QPN & SCEN & & QDN & SCEN \\
\hline Intercepto & -2984160 & -2988381 & PCLR & 0.65 & -0.65 \\
\hline PNR5L & 128.22 & 128.93 & PCTR & -0.3 & 0.3 \\
\hline PLR4L & -15.28 & -15.37 & INGPR & 110.54 & -110.54 \\
\hline PMR5L & -2.55 & -22.67 & $P O B$ & 0.01 & -0.01 \\
\hline PTR5L & -10.69 & -10.75 & QDNL & 0.57 & -0.57 \\
\hline PMZRL & 21.16 & 21.27 & PNRL & 0 & 0 \\
\hline PFRL & 9.83 & 9.88 & PENR & 0.11 & -0.11 \\
\hline PCHRL & -4.93 & -4.96 & PINR & -0.17 & 0.17 \\
\hline PSRL & 78.31 & 78.74 & PMNRL & -0.18 & 0.18 \\
\hline$S M G R$ & -6881.27 & -6919.38 & $D$ & 579.07 & -579.07 \\
\hline FERTR & -55.38 & -55.69 & PCNRL & -0.02 & 0.02 \\
\hline$P P$ & -89.23 & -89.72 & & & \\
\hline TEMP & 185270.5 & 186296.7 & & & \\
\hline QPNL & 0.67 & 0.67 & & & \\
\hline
\end{tabular}

Fuente: elaboración propia con datos de salida del modelo.

En la tabla 5 se presentan los valores medios de las variables que se utilizan en el modelo y que se emplean para obtener las elasticidades.

Tabla 5. Valor promedio de las variables usadas para el cálculo de las elasticidades

\begin{tabular}{|c|c|c|c|}
\hline Oferta & Periodo (2016-2018) & Demanda & Periodo (2016-2018) \\
\hline QPN & 2368061.7 & QDN & 145241.2 \\
\hline PNR5L & 1667.3 & PCNR & 13579.4 \\
\hline PLR4L & 4024.3 & PCLR & 16845.4 \\
\hline PMR5L & 1183.4 & PCTR & 15700.4 \\
\hline PTR5L & 3610.8 & INGPR & 130.8 \\
\hline PMZRL & 4397.9 & POB & 7686261.7 \\
\hline PFRL & 14450.3 & QDNL & 142807.7 \\
\hline PCHRL & 12496.9 & \multicolumn{2}{|c|}{ Transmisión de precios } \\
\hline PSRL & 5040.6 & PNR & 1733.36 \\
\hline SMGR & 87.4 & PMNR & 6817.95 \\
\hline FERTR & 7301.3 & PNRL & 1638.31 \\
\hline$P P$ & 1593.6 & PENR & 7482.02 \\
\hline TEMP & 23.2 & PINR & 9133.64 \\
\hline QPNL & 2328012 & PMNRL & 5560.96 \\
\hline \multicolumn{2}{|c|}{ Saldo de comercio } & $D$ & 1 \\
\hline SCEN & 2222820.43 & PCNRL & 11632.21 \\
\hline
\end{tabular}

Fuente: elaboración propia con datos de salida del modelo. 
Resultados económicos del modelo

En la tabla 6 se muestra la elasticidad precio propia de la demanda y la oferta. Además, también se presentan las elasticidades en relación con la transmisión de precios del mercado de la naranja en el estado de Veracruz. Los coeficientes de elasticidad precio de la demanda y oferta son de -0.03 y 0.09 , respectivamente. Ambos coeficientes indican una elasticidad inelástica, lo cual indica a su vez que si el precio promedio real al consumidor aumenta en $10 \%$, la cantidad demandada caerá en $0.3 \%$. Sin embargo, si el precio medio rural real aumenta en $10 \%$, la cantidad ofrecida aumentará en $0.9 \%$. Estas elasticidades son congruentes con la teoría de la oferta y la demanda, debido a que los valores se encuentran entre 0 y 1 , lo cual indica que tienen una elasticidad precio inelástica. Por el lado de la demanda, reacciona menos que proporcional a cambios en su respectivo precio; por el lado de la oferta, por ser la naranja un cultivo de ciclo largo de producción, no reacciona de manera inmediata a su respectivo precio (García-Mata, González-Machorro, García-Sánchez, Mora-Flores, González-Estrada y Martínez-Damián, 2013; Tomek y Kaiser, 2014; Vázquez y Martínez, 2015).

Tabla 6. Elasticidad propia y de transmisión de precios del mercado de la naranja en Veracruz, 2016-2018

\begin{tabular}{|c|c|c|c|c|c|}
\hline \multirow{2}{*}{ Variables exógenas } & \multicolumn{5}{|c|}{ Variables endógenas } \\
\hline & QPN & QDN & PNR & PMNR & PCNR \\
\hline PNR5L & 0.09 & & & & \\
\hline PCNR & & -0.03 & & & \\
\hline PMNR & & & 0.39 & & 1.04 \\
\hline PNRL & & & 0.41 & & \\
\hline PENR & & & & -0.18 & \\
\hline PINR & & & & 0.36 & \\
\hline PMNRL & & & & 0.22 & \\
\hline PCNRL & & & & & 0.05 \\
\hline
\end{tabular}

Fuente: elaboración propia con datos de salida del modelo.

En referencia al efecto de la transmisión de precios en términos reales al productor y al consumidor, el coeficiente de elasticidad que relaciona el precio medio rural de la naranja con el precio medio rural con un periodo de rezago y el precio promedio al mayoreo es 0.41 y 0.39 respectivamente, lo cual quiere decir que si se considera un aumento en el precio medio rural con un periodo de rezago y en el precio promedio al mayoreo de $10 \%$, el precio medio rural aumentará en $4.1 \%$ y $3.9 \%$, respectivamente. El precio promedio al mayoreo de la naranja se relaciona con el precio de la exportación y de la importación, además del precio promedio al mayoreo con un periodo de rezago, los coeficientes 
de elasticidad entre estas variables son $0.18,0.36$ y 0.22 , respectivamente. $\mathrm{Si}$ se considera un aumento de $10 \%$ en estos últimos tres precios, el precio promedio al mayoreo tendrá los siguientes efectos: disminuirá en $1.8 \%$ si el precio de exportación aumenta, incrementará en $3.6 \%$ si el precio de importación aumenta, e incrementará en $2.2 \%$ si el precio promedio al mayoreo con un periodo de rezago aumenta. El coeficiente de elasticidad que relaciona el precio promedio al consumidor de naranja con esta misma variable pero con un periodo de rezago y el precio promedio al mayoreo es de 0.05 y 1.04 , lo cual indica que ante aumentos del precio promedio al consumidor con un periodo de rezago y el precio promedio al mayoreo en $10 \%$, el precio promedio al consumidor aumentará en $0.5 \%$ y $10.4 \%$, respectivamente. Se puede notar que los efectos del precio al mayoreo son mayores en el precio al consumidor que al productor. Esta situación podría ser atribuida principalmente a las diversas etapas de la comercialización; es decir, como parte del proceso que posibilita que la fruta llegue al consumidor final, ésta adquiere mayor valor agregado que se refleja en el precio al consumidor (Ledesma, 2017).

Tabla 7. Elasticidades relacionadas con otros factores que afectan la oferta, la demanda y el saldo de comercio exterior de la naranja en Veracruz, 2016-2018

\begin{tabular}{|l|c|c|c|}
\hline \multirow{2}{*}{ Variables exógenas } & \multicolumn{3}{|c|}{ Variables endógenas } \\
\hline & QPN & QDN & SCEN \\
\hline PLR4L & -0.03 & & -0.03 \\
\hline PMR5L & -0.01 & & -0.01 \\
\hline PTR5L & -0.02 & & -0.02 \\
\hline PMZRL & 0.04 & & 0.04 \\
\hline PFRL & 0.06 & & 0.06 \\
\hline PCHRL & -0.03 & & -0.03 \\
\hline PSRL & 0.17 & & 0.18 \\
\hline SMGR & -0.25 & & -0.27 \\
\hline FERTR & -0.17 & & -0.18 \\
\hline PP & -0.06 & & -0.06 \\
\hline TEMP & 1.82 & & 1.94 \\
\hline QPNL & 0.65 & & 0.70 \\
\hline PCLR & & & -0.005 \\
\hline PCTR & & & 0.07 \\
\hline INGPR & & -0.03 & -0.01 \\
\hline POB & & 0.09 & -0.03 \\
\hline QDNL & & 0.44 & -0.04 \\
\hline FUEn: & & 0.51 & \\
\hline
\end{tabular}

Fuente: elaboración propia con datos de salida del modelo. 
En la tabla 7 se presentan los coeficientes de elasticidad que se relacionan con la oferta, la demanda y el saldo de comercio exterior de la naranja en el estado de Veracruz. Debido a que son demasiados coeficientes, sólo se destacan los de mayor magnitud. En el caso de los coeficientes que se relacionan con la oferta, los de mayor impacto son la temperatura (1.82), la cantidad producida de naranja con un año de rezago (0.65), el salario mínimo general (-0.25), el precio promedio del fertilizante $(-0.17)$ y el precio medio rural real de la sandía (0.17). Por el lado de la demanda, los coeficientes de elasticidad de mayor magnitud son cantidad demandada de naranja con un periodo de rezago $(0.51)$, la población $(0.44)$ y el ingreso per cápita real $(0.09)$. En este mismo tenor, los coeficientes de elasticidad que se relacionan con el saldo de comercio exterior son la temperatura (1.94), la cantidad producida de naranja con un periodo de rezago $(0.70)$, el salario mínimo general $(-0.27)$ y el precio promedio del fertilizante $(-0.18)$.

Si se considera el coeficiente de elasticidad de las siguientes variables: la temperatura, la cantidad producida de naranja con un periodo de rezago, el salario mínimo general, el precio promedio del fertilizante y el precio medio rural de la sandía, y un aumento en términos reales de $10 \%$ en dichas variables, la oferta experimentará los siguientes cambios: incrementa $18.2 \%$ si la temperatura aumenta, incrementa $6.5 \%$ si la cantidad ofrecida con un periodo de rezago aumenta, disminuye $2.5 \%$ si el salario mínimo general aumenta, disminuye $1.7 \%$ si el precio promedio del fertilizante aumenta, e incrementa $1.7 \%$ si el precio medio rural de la sandía aumenta. Estos resultados señalan que la oferta de la naranja en Veracruz depende de factores climáticos, como la temperatura (variable aleatoria). Considerando la ubicación geográfica de esta entidad en la república mexicana, es vulnerable a condiciones climáticas adversas (SAGARPA, 2015), lo cual implicaría que la oferta se vería mermada de manera considerable. También es posible apreciar que los factores relacionados con los costos de producción sí determinan de manera importante la oferta de la naranja. Esto significa que aumentar los costos de producción tendría efectos negativos sobre la producción.

Del mismo modo, para la demanda: aumenta $4.4 \%, 5.1 \%$ y $0.9 \%$ si la población, la cantidad demandada de naranja con un periodo de rezago y el ingreso per cápita real aumentan en $10 \%$. Estos resultados tienen sentido e implican que ante el crecimiento constante de la población es lógico pensar que se demandarían más alimentos, entre ellos fruta.

De acuerdo con la magnitud de los coeficientes de elasticidad que se relacionan con el saldo de comercio exterior de la naranja en el estado de Veracruz, son la temperatura (1.94), la cantidad producida con un periodo de rezago (0.70), el salario mínimo general $(-0.27)$ y el precio promedio del fertilizante $(-0.18)$. Si se toma en cuenta un aumento en términos reales de $10 \%$ en estos factores y considerando la magnitud de los coeficientes de elasticidad, el saldo de comercio exterior de la naranja en dicha entidad responderá de la siguiente manera: incrementa $19.4 \%$ si la temperatura aumenta, incrementa $7.0 \%$ si la cantidad producida con un periodo de rezago aumenta, disminuye $2.7 \%$ si el salario mínimo general aumenta y disminuye $1.8 \%$ si el precio promedio del 
fertilizante aumenta. El clima desempeña un papel importante para la producción de naranja, pues la variabilidad de ésta puede tener efectos favorables o desfavorables en el rendimiento de los cítricos (Rodríguez y Mendoza, 2014). En este mismo sentido, si se subsidiara el precio del fertilizante, como en el caso de los cultivos básicos, se podrían tener efectos positivos sobre la producción y por ende en el comercio exterior.

En la tabla 8 se muestra el efecto de los distintos escenarios de política económica sobre el mercado de la naranja en el estado de Veracruz. En el escenario 1 se puede observar la calibración del modelo, el cual es satisfactorio, dado que hay poca diferencia porcentual (menos de 10\%) entre los valores observados y los que predice el modelo tocante a las variables endógenas, a excepción del precio promedio real de la naranja al mayoreo, pues se encontró que el modelo está subestimando los valores observados en $16.8 \%$.

En el escenario 2 se analiza la política del gobierno que consiste en aumentar los salarios de 87.4 a 96.2 pesos diarios (promedio en términos reales de 2016 a 2018) equivalente a 10\% y los efectos de esta medida sobre las principales variables del mercado de la naranja en Veracruz, ceteris paribus. Los efectos de esta política hacen que la oferta se reduzca $2.5 \%$ (60.2 miles de t); en consecuencia, el precio medio rural real de la naranja disminuye 3.2\% (56.1 $\$ / t$ ), y debido a que existe una relación funcional directa entre el precio promedio real al mayoreo con el precio medio rural real y el precio promedio real al consumidor del cítrico, el precio promedio al mayoreo cae $9.9 \%(562.9 \$ / \mathrm{t})$ y el precio al consumidor declina 9.2\% (1 $161.0 \$ / \mathrm{t})$ referente al modelo base. La demanda se mantiene casi intacta, pues aumenta ligeramente $0.2 \%$ ( 0.3 miles de t), porque el precio al consumidor cayó. El efecto sobre el saldo de comercio exterior de la naranja en la entidad bajará 2.7\% (60.5 miles de t).

En el escenario 3 se analiza un aumento de $20 \%$ en el salario mínimo al pasar de 87.4 a 104.9 pesos diarios (promedio en términos reales de 2016 a 2018), ceteris paribus. Esta medida acentúa los efectos antes mencionados, la cantidad producida baja 5.1\% (120.3 miles de t), el precio medio rural, al mayoreo y al consumidor (en términos reales), cae 6.3\% (112.1\$/t), 19.9\% (1 $125.9 \$ / \mathrm{t})$ y $18.4 \%$ (2 $322.0 \$ / \mathrm{t})$, en cuanto al modelo base. La demanda no responde de manera importante, pues apenas aumenta 0.5\% (0.7 miles de $\mathrm{t})$, y el saldo de comercio exterior baja 5.4\% (121.0 miles de t).

En el escenario 4 se pretende analizar la política emprendida por el gobierno para dotar de fertilizantes a los actores rurales (subsidio); por lo tanto, en este escenario se simula dicha política disminuyendo el precio por tonelada del fertilizante en 10\%, al pasar de 7301.3 a $6571.1 \$ / t$ (promedio en términos reales de 2016 a 2018), ceteris paribus. Los efectos de esta medida son los siguientes: la cantidad producida aumenta 1.7\% (40.4 miles de t), el precio medio rural, al mayoreo y al consumidor (en términos reales), incrementa 2.1\% (37.7 $\$ / \mathrm{t})$, $6.7 \%(378.3 \$ / \mathrm{t})$ y $6.2 \%(780.1 \$ / \mathrm{t})$ en atención al modelo base. La demanda disminuye $0.2 \%$ ( 0.2 miles de $\mathrm{t})$ y el saldo de comercio exterior aumenta $1.8 \%$ (40.7 miles de $\mathrm{t})$.

En el escenario 5 se considera una disminución del precio por tonelada del fertilizante en $20 \%$, al pasar de 7301.3 a $5841.0 \$ / t$ (promedio en términos 
Tabla 8. Efecto de los costos de producción sobre el mercado de la naranja en Veracruz, 2016-2018

\begin{tabular}{|c|c|c|c|c|c|c|}
\hline Variables endógenas & QPN & QDN & PNR & PMNR & PCNR & SCEN \\
\hline Unidad de medida & Miles de $t$ & Miles de $\mathrm{t}$ & pesos/t & pesos/t & pesos/t & Miles de $\mathrm{t}$ \\
\hline $\begin{array}{l}\text { Situación observada } \\
(2016-2018)\end{array}$ & 2368.1 & 145.2 & 1733.4 & 6817.9 & 13579.4 & 2222.8 \\
\hline $\begin{array}{l}\text { Escenario 1. Modelo base } \\
(2016-2018)\end{array}$ & 2370.5 & 145.6 & 1773.2 & 5671.8 & 12610.8 & 2224.9 \\
\hline Cambio en porcentaje & $0.1 \%$ & $0.2 \%$ & $2.3 \%$ & $-16.8 \%$ & $-7.1 \%$ & $0.1 \%$ \\
\hline \multicolumn{7}{|l|}{ Escenarios } \\
\hline $\begin{array}{l}\text { Escenario 2. SMGR } \\
\text { aumenta } 10 \%\end{array}$ & 2310.3 & 145.9 & 1717.1 & 5108.9 & 11449.8 & 2164.4 \\
\hline $\begin{array}{l}\text { Escenario 3. SMGR } \\
\text { aumenta } 20 \%\end{array}$ & 2250.2 & 146.2 & 1661.1 & 4546.0 & 10288.8 & 2103.9 \\
\hline $\begin{array}{l}\text { Escenario 4. FERTR } \\
\text { disminuye } 10 \%\end{array}$ & 2410.9 & 145.3 & 1810.9 & 6050.1 & 13390.9 & 2265.6 \\
\hline $\begin{array}{l}\text { Escenario 5. FERTR } \\
\text { disminuye } 20 \%\end{array}$ & 2451.4 & 145.1 & 1848.5 & 6428.4 & 14171.1 & 2306.2 \\
\hline $\begin{array}{l}\text { Escenario 6. SMGR aumenta } \\
10 \% \text { y FERTR disminuye } 10 \%\end{array}$ & 2350.8 & 145.7 & 1754.8 & 5487.2 & 12229.9 & 2205.1 \\
\hline $\begin{array}{l}\text { Escenario 7. SMGR aumenta } \\
20 \% \text { y FERTRdisminuye } 20 \%\end{array}$ & 2331.0 & 145.8 & 1736.4 & 5302.5 & 11849.1 & 2185.2 \\
\hline \multicolumn{7}{|l|}{$\begin{array}{l}\text { Cambio respecto } \\
\text { al modelo base }\end{array}$} \\
\hline $\begin{array}{l}\text { Escenario 2. SMGR } \\
\text { aumenta 10\% }\end{array}$ & -60.2 & 0.3 & -56.1 & -562.9 & -1161.0 & -60.5 \\
\hline $\begin{array}{l}\text { Escenario 3. SMGR } \\
\text { aumenta } 20 \%\end{array}$ & -120.3 & 0.7 & -112.1 & -1125.9 & -2322.0 & -121.0 \\
\hline $\begin{array}{l}\text { Escenario 4. FERTR } \\
\text { disminuye } 10 \%\end{array}$ & 40.4 & -0.2 & 37.7 & 378.3 & 780.1 & 40.7 \\
\hline $\begin{array}{l}\text { Escenario 5. FERTR } \\
\text { disminuye } 20 \%\end{array}$ & 80.9 & -0.4 & 75.3 & 756.5 & 1560.3 & 81.3 \\
\hline $\begin{array}{l}\text { Escenario 6. SMGR aumenta } \\
10 \% \text { y FERTR disminuye } 10 \%\end{array}$ & -19.7 & 0.1 & -18.4 & -184.7 & -380.9 & -19.8 \\
\hline $\begin{array}{l}\text { Escenario 7. SMGR aumenta } \\
20 \% \text { y FERTR disminuye } 20 \%\end{array}$ & -39.5 & 0.2 & -36.8 & -369.3 & -761.7 & -39.7 \\
\hline \multicolumn{7}{|l|}{$\begin{array}{l}\text { Cambio en porcentaje } \\
\text { respecto al modelo base }\end{array}$} \\
\hline $\begin{array}{l}\text { Escenario 2. SMGR } \\
\text { aumenta } 10 \%\end{array}$ & -2.5 & 0.2 & -3.2 & -9.9 & -9.2 & -2.7 \\
\hline $\begin{array}{l}\text { Escenario 3. SMGR } \\
\text { aumenta } 20 \%\end{array}$ & -5.1 & 0.5 & -6.3 & -19.9 & -18.4 & -5.4 \\
\hline $\begin{array}{l}\text { Escenario 4. FERTR } \\
\text { disminuye } 10 \%\end{array}$ & 1.7 & -0.2 & 2.1 & 6.7 & 6.2 & 1.8 \\
\hline $\begin{array}{l}\text { Escenario 5. FERTR } \\
\text { disminuye } 20 \%\end{array}$ & 3.4 & -0.3 & 4.2 & 13.3 & 12.4 & 3.7 \\
\hline $\begin{array}{l}\text { Escenario 6. SMGR aumenta } \\
20 \% \text { y FERTR disminuye } 10 \%\end{array}$ & -0.8 & 0.1 & -1.0 & -3.3 & -3.0 & -0.9 \\
\hline $\begin{array}{l}\text { Escenario 7. SMGR aumenta } \\
20 \% \text { y FERTR disminuye } 20 \%\end{array}$ & -1.7 & 0.2 & -2.1 & -6.5 & -6.0 & -1.8 \\
\hline
\end{tabular}

Fuente: elaboración propia con datos de salida del modelo. 
reales de 2016 a 2018), ceteris paribus. Los efectos que trae consigo esta medida son que la cantidad producida aumenta 3.4\% (80.9 miles de $\mathrm{t}$ ), el precio medio rural, al mayoreo y al consumidor (en términos reales), incrementa $4.2 \%$ $(75.3 \$ / \mathrm{t}), 13.3 \%(756.5 \$ / \mathrm{t})$ y $12.4 \%(1560.3 \$ / \mathrm{t})$ con respecto al modelo base. La demanda disminuye $0.3 \%$ ( 0.4 miles de $\mathrm{t})$ y el saldo de comercio exterior sube $3.7 \%$ ( 81.3 miles de $\mathrm{t})$.

En el escenario 6 se analiza un cambio simultáneo en los costos de producción de la naranja en Veracruz; es decir, se considera que el salario mínimo aumenta en términos reales en $10 \%$ y el precio promedio del fertilizante disminuye en $10 \%$, ceteris paribus. Esto origina que la cantidad producida disminuya 0.8\% (19.7 miles de $\mathrm{t}$ ), el precio medio rural, al mayoreo y al consumidor (en términos reales), cae $1 \%(18.4 \$ / \mathrm{t}), 3.3 \%(184.7 \$ / \mathrm{t})$ y $3.0 \%(380.9 \$ / \mathrm{t})$ en relación con el modelo base. La demanda aumenta $0.1 \%(0.1$ miles de $t)$ y el saldo de comercio exterior disminuye $0.9 \%$ (19.8 miles de $\mathrm{t}$ ).

En el escenario 7, al igual que en el anterior, se considera un aumento en salario mínimo y una disminución en el precio promedio del fertilizante (en términos reales) de $20 \%$, ceteris paribus. Los efectos de esta medida son que la cantidad producida disminuye $1.7 \%$ (39.5 miles de $\mathrm{t}$ ), el precio medio rural, al mayoreo y al consumidor (en términos reales), cae $2.1 \%$ (36.8 $\$ / \mathrm{t}), 6.5 \%(369.3$ $\$ / \mathrm{t})$ y $6.0 \%(761.7 \$ / \mathrm{t})$ en cuanto al modelo base. La demanda aumenta $0.2 \%$ (0.2 miles de $\mathrm{t}$ ) y el saldo de comercio exterior declina $1.8 \%$ (39.7 miles de $\mathrm{t}$ ).

Después de analizar los distintos escenarios, se puede percibir que aumentar los costos de producción no es recomendable para el mercado de la naranja en Veracruz, pues un aumento en el salario mínimo en términos reales afecta negativamente la producción, el precio medio rural real y el precio promedio real al mayoreo, así como el saldo de comercio exterior. Esto perjudica el ingreso porque baja el precio medio rural de la naranja. En otras palabras, disminuye el precio de venta de la fruta y podría mermar la calidad de vida de los citricultores (productores) en Veracruz. También se sabe que dicha entidad tiene excedentes considerables de este cítrico, pero incrementar el salario hará que bajen dichos excedentes, lo cual puede traducirse en pérdida de competitividad en la exportación del cítrico a otras entidades del país o incluso a escala internacional. La medida antes mencionada sólo favorece el precio promedio real al consumidor, porque éste disminuye considerablemente, pero debido a que existe una elasticidad precio de la demanda inelástica, la demanda casi permanece sin cambios al disminuir el precio al consumidor. Lo que sí es recomendable es disminuir los costos de producción (disminuir el precio del fertilizante), ya que esta medida hace que la producción incremente, así como los distintos precios, a excepción del precio al consumidor, que se incrementa y perjudica la demanda, pero debido a la elasticidad precio de la demanda muy inelástica no perjudica de manera importante (demanda estable). El saldo de comercio exterior crece y permite que el estado de Veracruz gane competitividad en el cultivo de la naranja. Esto permitiría que exportara más cantidad de este cítrico a escala nacional e internacional o bien podría destinarlo a la agroindustria presente en la entidad. En la actualidad, según Martínez (2019), sólo se exporta $1 \%$ de la naranja, principalmente hacia Estados Unidos. Así mis- 
mo se podría aprovechar la industria procesadora de jugos en México (24 plantas), pues ésta apenas demanda $20 \%$ del total de la producción de la naranja nacional. Dicha agroindustria se concentra en Tamaulipas, Nuevo León, San Luís Potosí, Tabasco, Puebla y Yucatán, lugares cercanos al principal estado productor. Esta medida es factible, dado que hay mucha industria y poca demanda de insumo (naranja).

Al analizar un cambio de manera simultánea (aumento en el salario mínimo y la disminución en el precio promedio del fertilizante), se puede notar que los efectos, aunque mínimos, son perjudiciales en la producción, en los precios y en el saldo de comercio exterior de la naranja en Veracruz (véase tabla 8). Esto sucede porque el efecto del salario mínimo es superior al de la disminución del precio promedio del fertilizante sobre la producción de naranja.

\section{Conclusiones}

La elasticidad precio de la oferta y la demanda de la naranja en el estado de Veracruz responde de manera inelástica a sus respectivos precios, debido a que es un cultivo perenne y la reacción ante cambios en los precios no es inmediata.

Los factores que determinan en mayor medida el comportamiento de la oferta de la naranja en Veracruz son la temperatura, la cantidad producida con un periodo de rezago, el salario mínimo general en términos reales, el precio promedio real del fertilizante y el precio medio rural real de la sandía. En este mismo sentido, por el lado de la demanda, los factores que la determinan son la cantidad demandada con un periodo de rezago, la población y el ingreso per cápita real.

El sistema de ecuaciones simultáneas y los escenarios propuestos permitieron observar que los costos de producción (salario mínimo y el precio promedio del fertilizante) desempeñan un papel decisivo en el mercado de la naranja en el estado de Veracruz, pues determinan la producción, la demanda, los precios y el saldo de comercio exterior del cítrico.

Los distintos escenarios de política sugirieron que no es recomendable aumentar los costos de producción, porque originan que la oferta de la naranja disminuya, y viceversa.

Se encontró que la demanda de la naranja en el estado de Veracruz es muy estable, y no sufre cambios de manera significativa por el aumento o disminución en los costos de producción. Por tal razón, se recomienda al gobierno federal ampliar el programa de subsidio a los fertilizantes para que se incluyan otros cultivos, como los cítricos (naranja, limón, mandarina y toronja), y moderar los aumentos en el salario mínimo, con la finalidad de poder beneficiar a los citricultores del estado de Veracruz, aumentando la producción, el precio medio rural real y el saldo de comercio exterior, para permitir a los citricultores exportar más fruta hacia otras entidades del país, o incluso al extranjero, o bien enviar los excedentes de naranja a las plantas procesadoras de jugo (agroindustria). Todo esto podría mejorar los ingresos y la calidad de vida de los citricultores veracruzanos. 


\section{Referencias}

Almaguer-Vargas, G., y Ayala-Garay, A. V. (2014). Adopción de innovaciones en limón "persa" (Citrus latifolia Tan) en Tlapacoyan, Veracruz. Revista Chapingo Serie Horticultura, 20(1), 89-100. doi: 10.5154/r.rchsh.2010.10.076

Atucha, A. J., y Gualdoni, P. (2018). El funcionamiento de los mercados. Material de cátedra de introducción a la economía. Recuperado de http://nulan. mdp.edu.ar/2879/1/atucha-etal-2018.pdf

Aznar Sánchez, J. A., Pérez Mesa, J. C., y Galdeano Gómez, E. (2015). Análisis del sector citrícola español. Almería: Cajamar Caja Rural. Recuperado de http: / / www.besana.es/sites/default/files/analisis-del-sector-citricola-espanol-2.pdf

Banco de México (Banxico). (2019). Mercado cambiario. Recuperado de http:// www.banxico.org. mx/portal-mercado-cambiario/

Barkley, A., y Barkley, P. W. (2013). Principles of agricultural economics. Nueva York: Routledge.

Barrera-Rodríguez, A. I., Jaramillo-Villanueva, J. L., Escobedo-Garrido, J. S., y Herrera-Cabrera, B. E. (2011). Rentabilidad y competitividad de los sistemas de producción de vainilla (Vanilla planifolia J.) en la región del Totonacapan, México. Agrociencia, 45(5), 625-638. Recuperado de https://www. colpos.mx/agrocien/Bimestral/2011/jul-ago/jul-ago-11.html

Barrón Pérez, M. A., y Hernández Trujillo, J. M. (2014). Productores y cortadores de naranja, una relación fallida. El municipio de Álamo Temapache, Veracruz. Revista Mexicana de Ciencias Agrícolas, 5(6), 1097-1109.

Caamal Cauich, I., Jerónimo Ascencio, F., y Pat Fernández, V. G. (2016). Análisis de las variables económicas de la producción de naranja en México. IV Congreso Internacional y XV Congreso Nacional de Investigación y Servicio Territorio, sociedad, desarrollo y ambiente. Enfoque interdisciplinario. Texcoco, Estado de México. Revista Mexicana de Ciencias Agrícolas, 153-162. Recuperado de https://cienciasagricolas.inifap.gob.mx/index.php/publicaciones/ ponencias/35-iv-congreso-internacional-y-xv-congreso-nacional-de-investigacion-y-servicio-territorio-sociedad-desarrollo-y-ambiente-enfoque-interdiciplinario

Cárdenas Ramírez, J. R. (2006). La citricultura en Veracruz. CEDEFRUT A. C. Recuperado de http://www.concitver.com/15_9citricultura.html

Comisión Nacional de Salarios Mínimos (CONASAMI). (2019). Salario mínimo general promedio de los Estados Unidos Mexicanos 1964-201. Recuperado de https://www.gob.mx/cms/uploads/attachment/file/105354/Salario_Minimo_General_Promedio_de_los_Estados_Unidos_Mexicanos_1964_-_2016.pdf

Consejo Nacional de Población (CONAPO). (2019). Proyecciones de la población de México y de las entidades federativas, 2016-2050. Recuperado de https://datos.gob.mx/busca/dataset/proyecciones-de-la-poblacion-de-mexico-y-de-las-entidades-federativas-2016-2050

Diario Oficial de la Federación. (2019). Acuerdo por el que se emiten los lineamientos de operación del programa de fertilizantes para el ejercicio fiscal 2019. Recuperado de https://www.gob.mx/cms/uploads/attachment/ 
file/447318/Lineamientos_de_Operaci_n_del_Programa_de_Fertilizantes_2019.pdf

Fideicomisos Instituidos en Relación con la Agricultura (FIRA). (2018). Costos de producción. Recuperado de https://www.fira.gob.mx/Nd/Agrocostos.jsp

Franco Sánchez, M. A., Leos Rodríguez, J. A., Salas González, J. M., Acosta Ramos, M. , y García Munguía, A. (2018). Análisis de costos y competitividad en la producción de aguacate en Michoacán, México. Revista Mexicana de Ciencias Agrícolas, 9(2), 391-403. doi: 10.29312/remexca.v9i2.1080

García-Mata, R., González-Machorro, M. F., García-Sánchez, R. C., Mora-Flores, J. S., González-Estrada, A., y Martínez-Damián, M. A. (2013). El Mercado del plátano (Musa paradisiaca) en México, 1971-2017. Agrociencia, 47(4), 399-410. Recuperado de https://www.colpos.mx/agrocien/Bimestral/2013/ may-jun/may-jun-13.html

Gómez Cruz, M. A., y Schwentesius Rindermann, R. (1997). La Agroindustria de naranja en México. Estado de México: CIESTAAM.

Gujarati, D. N., y Porter, D. C. (2010). Econometría. México: McGraw-Hill.

Hernández, G. (17 de diciembre de 2019). AMLO celebra el aumento al salario mínimo: sube a 123.22 en 2020. Vanguardia. Recuperado de https://vanguardia.com.mx/articulo/amlo-celebra-el-aumento-al-salario-minimo-sube-12322-en-2020

Hernández Torres, N. (2011). La comercialización de la naranja (Citrus x sinensis) del municipio de Yahualica, Hidalgo (tesis de licenciatura). Recuperada de http://repositorio.uaaan.mx:8080/xmlui/bitstream/handle/123456789/5272/T18887\%20HERNANDEZ\%20TORRES\%2C\%20NORMA\%20 \%20\%20TESIS.pdf?sequence=1\&isAllowed=y

Instituto Nacional de Estadística y Geografía (INEGI). (2019a). Banco de Información Económica. Recuperado de https://www.inegi.org.mx/app/indicadores $/$ ?tm $=0$

Instituto Nacional de Estadística y Geografía (INEGI). (2019b). Precios. Recuperado de https: / / www.inegi.org. $\mathrm{mx} /$ datos $/$ ?t=0210

Ledesma Jiménez, M. A. (2017). Valoración de los canales de comercialización del rubro naranja (Citrus sinensis L), en el Cantón Caluma provincia Bolívar (tesis de licenciatura). Recuperada de http://dspace.ueb.edu.ec/bitstream/123456789/1855/1/VALORACI\%C3\%93N\%20DE\%20LOS\%20CANALES\%20 PDJ.pdf

López-Hernández, W. A., Garza-Bueno, L. E., Cruz-Galindo, B., y Nieto-Ángel, R. (2019). Competitividad del limón persa en la región del Papaloapan, Oaxaca. Revista Mexicana de Ciencias Agrícolas, 10(4), 921-934. doi: 10.29312/ remexca.v10i4.408

López Santiago, M. A., Valdivia Alcalá. R., Hernández Ortiz, J., y Romo Lozano, J. L. (2010). Elasticidades y flexibilidades de los productos citrícolas en México. Revista Mexicana de Economía Agrícola y de los Recursos Naturales, 3(2), 97-112. Recuperado de https://www.chapingo.mx/revistas/remecaren/contenido.php?id_revista_numero=121

Martínez Jiménez, A. (2019). Planeación de la producción de naranja en México y análisis de la volatilidad de precios (tesis de doctorado). Recuperada de 
http://colposdigital.colpos.mx:8080/jspui/bitstream/handle/10521/3663/ Martinez_Jimenez_A_DC_ISEl_Economla_2019.pdf?sequence=1\&isAllowed=y Molina, N. A. (2016). Costos y rentabilidad citrícola: capacitación a productores de cambio rural II. Hoja de divulgación número 45. Instituto Nacional de Tecnología Agropecuaria. Ministerio de Agroindustria de la Nación. Bella vista, Corrientes, Argentina. Recuperado de https: //inta.gob.ar/sites/default/ files/inta_hd45_citrus_cambiorural.pdf

Moreno Tapia, H. (18 de noviembre de 2017). El campo, con sabor a naranja. La Opinión de Poza Rica. Recuperado de https://www.laopinion.net/campo-sabor-naranja/

Nicholson, W. (2008). Teoría microeconómica. Principios básicos y ampliaciones. México: Cengage Learning.

Ochoa Neira, M. G. (16 de noviembre de 2015). Importancia de los costos de cultivo (I). El Economista. Recuperado de https://www.eleconomista.com. $\mathrm{mx/opinion/Importancia-de-los-costos-de-cultivo-I-20151116-0006.html}$

Panorama Agroalimentario. (2019). Servicio de Información Agroalimentaria y Pesquera. Recuperado de https://nube.siap.gob.mx/gobmx_publicaciones_ siap/pag/2019/Atlas-Aagoalimentario-2019

Pat-Fernández, V., Caamal-Cauich, I., Jerónimo-Ascencio, F., y Mendoza-Tornez, R. (2015). Costos y competitividad de la producción del limón persa en el municipio de Martínez de la Torre, Veracruz. En F. Pérez, E. Figueroa, y L. Godínez (coords.), Ciencias Sociales: Economía y Humanidades. Handbook T-I. (pp. 295-308). México: ECOFARM. Recuperado de http:// ri.uaemex.mx/handle/20.500.11799/41208

Ramírez Abarca, O., Figueroa Hernández, E., y Espinosa Torres, L. E. (2015). Análisis de rentabilidad de la tuna en los municipios de Nopaltepec y Axapusco, Estado de México. Revista Mexicana de Agronegocios, 36, 199-1210. Recuperado de https: / / www.redalyc.org/articulo.oa?id=14132408006

Rebollar Rebollar, S., Hernández Martínez, J., Guzmán Soria, E., Morales Hernández, J. L., y Rebollar Rebollar, A. (2014). Costos de producción de maíz de temporal en una región campesina del oriente de Michoacán. Pistas Educativas, 34(107), 47-60. Recuperado de http://www.itcelaya.edu.mx/ojs/ index.php/pistas/article/view/1275/1084

Rodríguez Quibrera, C. G., y Mendoza Herrera, A. (2014). Una amenaza para la citricultura mexicana. La ciencia y el Hombre, 27(1). Recuperado de https://www.uv.mx/cienciahombre/revistae/vol27num1/contenido.html

Secretaría de Agricultura, Ganadería, Desarrollo Rural, Pesca y Alimentación (SAGARPA). (2015). Agenda técnica agrícola de Veracruz. Recuperado de https://issuu.com/senasica/docs/30_veracruz_2015_sin

Secretaría del Medio Ambiente y Recursos Naturales (SEMARNAT). (2019). Precipitación media por entidad federativa (milímetros). Recuperado de http://dgeiawf.semarnat.gob.mx:8080/ibi_apps/WFServlet?IBIF_ex=D3_ AGUA01_09\&IBIC_user=dgeia_mce\&IBIC_pass=dgeia_mce\&NOMBREENTIDA$D={ }^{*} \& N O M B R E A N I O=*$ 
Servicio de Información e Integración de Mercados (SNIIM). (2019a). Anuario estadístico de mercados nacionales. Insumos agrícolas. Recuperado de http:/ / www.economia-sniim.gob.mx/SNIIM-AN/estadisticas/e_anuariosinsu.asp?

Servicio de Información e Integración de Mercados (SNIIM). (2019b). Anuario estadístico de mercados nacionales. Frutas y hortalizas. Recuperado de http: / / www.economia-sniim.gob.mx/SNIIM-AN/estadisticas/e_fyhAnuarioa.asp?

Servicio Meteorológico Nacional (SMN). (2019). Resúmenes mensuales de temperaturas y lluvia. Recuperado de https://smn.conagua.gob.mx/es/ climatologia/temperaturas-y-lluvias/resumenes-mensuales-de-temperaturas-y-lluvias

Sistema de Información Agroalimentaria y Pesquera (SIAP). (2019). Anuario estadístico de la producción agrícola. Recuperado de https://nube.siap.gob. $\mathrm{mx} /$ cierreagricola/

Soler Montcouquiol, R., y Hernández Plascencia, J. A. (2005). Producción y comercialización de la naranja: caso región Acateno Hueytamalco en el Estado de Puebla, México. Revista Mexicana de Agronegocios, 9(16), 510-519. Recuperado de https://www.redalyc.org/pdf/141/14101610.pdf

Statistical Analysis System Institute (SAS). (2013). SAS/ETS user's guide, version 9.2. SAS. NC: USA.

Statistics Division of Food and Agriculture Organization of the United Nations (FAOSTAT). (2019a). Archivos fertilizantes. Recuperado de http://www.fao. org/faostat/es/\#data/RA

Statistics Division of Food and Agriculture Organization of the United Nations (FAOSTAT). (2019b). Cultivos y productos de ganadería. Recuperado de http://www.fao.org/faostat/es/\#data/TP

Statistics Division of Food and Agriculture Organization of the United Nations (FAOSTAT). (2019c). Series anuales temporales. Recuperado de http://www. fao.org/faostat/es/\#data/OA

Tierra Fértil. (6 de marzo de 2019). Incosteable el precio de la naranja en Veracruz. Tierra Fértil. Recuperado de https://www.tierrafertil.com.mx/ incosteable-el-precio-de-la-naranja-en-veracruz/

Tomek, W. G., y Kaiser, H. M. (2014). Agricultural product prices. Nueva York: Cornell University Press.

Vázquez Alvarado, J. M. P., y Martínez Damián, M. A. (2015). Estimación empírica de elasticidades de oferta y demanda. Revista Mexicana de Ciencias Agrícolas, 6(5), 955-965. Recuperado de http://www.scielo.org.mx/scielo. php?script=sci_arttext\&pid=S2007-09342015000500004 